\title{
DEFINICIÓN DE UN PROTOCOLO PARA LA MEDIDA PRECISA DEL RANGO CERVICAL EMPLEANDO TECNOLOGÍA INERCIAL
}

\author{
Á. Martín ${ }^{1}$, R. Raya ${ }^{1,2}$, C. Sánchez ${ }^{1}$, R. García ${ }^{1}$, O. Ramírez ${ }^{2}$, A. Otero ${ }^{1}$ \\ ${ }^{1}$ Universidad San Pablo CEU, rafael.rayalopez@ceu.es \\ ${ }^{2}$ Werium Assistive Solutions SL, oscar.ramirez@weriumsolutions.com
}

\begin{abstract}
Resumen
Este artículo tiene por objetivo definir un protocolo para la medida precisa del rango articular cervical empleando la tecnología inercial. La utilización de instrumentos de medida, como los goniómetros tradicionales, la fotogrametría o los sensores inerciales permiten una medida objetiva para mejorar el diagnóstico de patologías y su rehabilitación. En este artículo se estudia cual es el mejor posicionamiento de dos sensores $e$ identificamos los factores que influyen en la medida precisa del rango cervical. En el estudio participaron sujetos sin dolor cervical. La medida angular en dos sesiones independientes, siguiendo el protocolo establecido, muestra una diferencia menor a un grado entre ellas, lo que demuestra ser un sistema y protocolo válidos para el objetivo definido al comienzo del trabajo.
\end{abstract}

Palabras Clave: Sensor inercial, cervical, biomecánica, goniometría.

\section{INTRODUCCIÓN}

El rango de movimiento cervical puede disminuir por muchas causas o condiciones: dolor articular, inflamación, infección, lesiones motoras o neuromotoras, [1]. Una de las causas con mayor prevalencia es el llamado latigazo o esguince cervical (whiphash), [2], una lesión provocada por un movimiento violento de flexo-extensión cervical. Alrededor de un $25 \%$ de los accidentes de tráfico provocan esta lesión, según la Dirección General de Tráfico (DGT). El 98\% de las lesiones cervicales están causadas por accidentes de tráfico.

Unida a esta lesión, podemos encontrar otras patologías, como es el caso de las lesiones neuromotoras, como por ejemplo, la Parálisis Cerebral, [3], (2-3 casos por cada 1000 habitantes) en el que es frecuente la hipotonía, un tono muscular debilitado que dificulta el mantenimiento de una postura cervical adecuada.

En todos estos casos se requiere una medida del rango articular para apoyar un diagnóstico correcto y para medir, de forma objetiva, el progreso terapéutico. Existen diversos instrumentos y dispositivos de medición, como la fotogrametría, los inclinómetros, los sensores inerciales o los goniómetros tradicionales. La tecnología inercial presenta interesantes ventajas debido a su simplicidad, bajo coste, precisión y la capacidad de registrar los resultados automáticamente.

La medida angular y su comparación con los considerados patrones normales del rango de movimiento cervical permiten medir la evolución de la lesión cuando el paciente recibe una terapia o un tratamiento. En este sentido, existen diversos artículos científicos en los que se establecen la normalidad del rango de movimiento cervical, [4, 5]. En estos artículos destaca la gran heterogeneidad en los resultados, que parecen poner de manifiesto que existe un rango de normalidad muy amplio. La Tabla 1 muestra los resultados de normalidad de dos sesiones consecutivas medidas en pacientes sanos, [4].

En nuestro estudio, participarán sujetos sanos en los que, en primer lugar estudiaremos la relación del rango cervical estimado con el que se presenta en la literatura científica. Realizaremos distintos protocolos con el fin de identificar cómo influyen principalmente el posicionamiento de los sensores en el cuerpo del sujeto y la importancia de definir correctamente la posición neutral en la que se calibra el sistema para realizar la medición del rango articular.

La determinación de la posición neutral es un factor clave y puede condicionar la repetitividad entre medidas de un mismo sujeto o una misma población. La hipótesis que se plantea en este artículo es que la medida del rango total del movimiento mejora la precisión respecto a la medida de los rangos independientes. Es decir, el movimiento a evaluar debe ser una flexoextensión completo (de extremo a extremo) en lugar de una flexión y una extensión independientes. Con el fin de ver si existen diferencias significativas entre ambos casos, el artículo presenta el protocolo I y II que miden los rangos de forma independiente $\mathrm{y}$ completa respectivamente.

Otro de los factores que influyen significativamente en la estimación angular, es la ubicación de los sensores en los segmentos corporales. En este sentido, existen algunos estudios que concluyen que la mejor ubicación para estimar el rango cervical es 
ubicar un sensor en C0 (cabeza) y otro sensor en la vértebra T4, [6].

Tabla 1: Rango Normal del Movimiento Cervical medido en dos sesiones consecutivas, [4]

\begin{tabular}{|l|l|l|}
\hline Movimiento & $\begin{array}{l}\text { Amplitud } \\
\text { Sesión1 }\end{array}$ & $\begin{array}{l}\text { Amplitud } \\
\text { Sesión 2 }\end{array}$ \\
\hline Flexión & $52,9^{\circ} \pm 9,0^{\circ}$ & $52,6^{\circ} \pm 7,1^{\circ}$ \\
\hline Extensión & $78,8^{\circ} \pm 12,5^{\circ}$ & $80,2^{\circ} \pm 13,4^{\circ}$ \\
\hline $\begin{array}{l}\text { Inclinación } \\
\text { derecha }\end{array}$ & $41,4^{\mathbf{o}} \pm 8,5^{\circ}$ & $42,1^{\mathbf{o}} \pm 9,5^{\circ}$ \\
\hline $\begin{array}{l}\text { Inclinación } \\
\text { izquierda }\end{array}$ & $47,0^{\circ} \pm 9,4^{\circ}$ & $46,6^{\circ} \pm 8,6^{\circ}$ \\
\hline $\begin{array}{l}\text { Rotación } \\
\text { derecha }\end{array}$ & $73,2^{\circ} \pm 7,2^{\circ}$ & $73,4^{\circ} \pm 7,5^{\circ}$ \\
\hline $\begin{array}{l}\text { Rotación } \\
\text { izquierda }\end{array}$ & $74,8^{\circ} \pm 9,7^{\circ}$ & $74,9^{\circ} \pm 9,0^{\circ}$ \\
\hline
\end{tabular}

\section{METODOLOGÍA}

\section{$2.1 \quad$ EQUIPOS}

Los equipos empleados han sido fabricados por la empresa Werium Assistive Solutions SL. Los sensores ofrecen, de acuerdo a la información proporcionada por el fabricante, una precisión de 1 grado, con una frecuencia de muestreo de $50 \mathrm{~Hz}$. Estos sensores se conectan de forma inalámbrica al computador mediante una conexión Bluetooth. En esta experimentación se emplean dos sensores con el fin de medir la orientación de los dos segmentos corporales que intervienen en el movimiento cervical: cabeza y tronco.

El software ofrecido por Werium permite la captura y generación de un informe con la medida articular realizada. Este informe será empleado para la extracción de los datos presentados en este artículo.

\section{$2.2 \quad$ PROTOCOLO}

\subsubsection{Participantes. Movimientos a realizar durante la medida del rango cervical}

En la experimentación participaron 36 sujetos (16 mujeres y 20 hombres) de 25,33 $\pm 8,6$ años de edad sin ninguna patología cervical para el Protocolo I. Para el protocolo II participaron 27 sujetos (10 mujeres y 17 hombres) con 26,63 \pm 9,44 años de edad sin ninguna patología cervical. Se realizó una evaluación estadística para determinar que no existe una diferencia significativa en cuanto a la edad entre ambos grupos.
La persona encargada de realizar la medición explicó al inicio de la experimentación la prueba a realizar. El sujeto debía realizar 6 movimientos de cabeza alcanzando la máxima amplitud en los tres ejes de movimiento, esto es, realizar una flexión, una extensión, una inclinación izquierda y derecha, una rotación izquierda y derecha. Los movimientos se realizaron siguiendo un orden aleatorio. El sujeto realizó los movimientos sentado en una silla, con las manos sobre las piernas, la espalda apoyada sobre el respaldo y las rodillas formando un ángulo recto con los pies completamente apoyados sobre el suelo.

Cada sujeto repitió los movimientos en dos sesiones independientes con el fin de estudiar la correlación y repetitividad entre ambas.

\subsubsection{Factores que incluyen en el protocolo de medida}

Como se ha comentado, con el fin de validar el protocolo para la medida del rango cervical se estudian dos factores considerados claves para alcanzar la máxima precisión y repetitividad: la ubicación de los sensores y el establecimiento de la posición neutral a partir de la que se evalúa el rango articular.

Para estudiar cómo influye la ubicación de los sensores se estudiarán dos opciones que han sido identificadas en la bibliografía como las más adecuadas, [6]. La primera opción será colocar el primer sensor en C0 (cabeza) y el segundo sensor en la vértebra $\mathrm{C} 7$. La segunda opción será colocar el primer sensor en $\mathrm{C} 0$ (cabeza) y el segundo en la vértebra $\mathrm{T} 4$. La figura 1 muestra una posible ubicación de los sensores utilizados.

El segundo factor que se estudia en este artículo es el efecto de la posición neutral, posición cero o posición en la que se calibran los dispositivos antes de iniciar la medida. Una variación en la posición neutral en medidas consecutivas puede obviamente modificar el rango de medición, lo que deteriora la repetitividad de la medida. Esto es particularmente importante si consideramos que este tipo de instrumentos será empleado para determinar si existe una mejora del rango articular ante una terapia o tratamiento.

Para verificar este hecho, realizamos dos protocolos. En el protocolo I, cada sujeto realizará los 6 movimientos de forma independiente y calibrando al inicio de cada uno de ellos, esto es: 1) Flexión, 2) Extensión, 3) Inclinación izquierda, 4) Inclinación derecha, 5) Rotación izquierda, 6) Rotación derecha. En este protocolo, el cálculo de la posición neutral se reinicia al comienzo de cada movimiento. Esto puede favorecer mayor variabilidad en los resultados medidos, debido a que la postura neutral puede variar de una medida a otra.

Para verificar el efecto de este factor se plantea el Protocolo II en el que se mide el rango total del movimiento de extremo a extremo, esto es: 1) 
Flexoextensión, 2) Inclinación derecha-izquierda y 3) Rotación izquierda-derecha. En este caso, el sistema se calibra al iniciar cada movimiento completo. De esta forma, el rango total no es tan dependiente de la posición neutral y, según nuestra hipótesis, se espera que mejore la repetitividad en la medida siguiendo el Protocolo II.

Una vez analizados los resultados de cada protocolo, se comparan entre las dos sesiones realizadas y con las publicaciones científicas que determinan el patrón normal del rango cervical, citadas en la sección anterior.

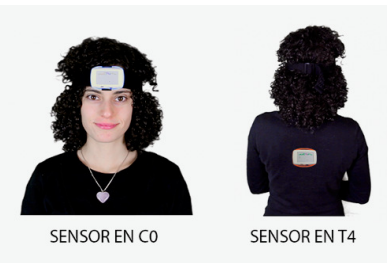

Figura 1. Ubicación de los sensores en C0 y T4

\subsubsection{Análisis de los resultados}

Para obtener los resultados de la medida articular se empleó el software de Werium. De la captura realizada se obtuvieron los rangos máximos y mínimos de cada movimiento, de los que finalmente se calculó la media y desviación para todos los usuarios para la sesión 1 y la sesión 2 (set 1 y set 2 en las figuras de la sección siguiente). Adicionalmente, se calculó el coeficiente de correlación intraclase (ICC) para determinar la fiabilidad de las medidas entre las dos sesiones realizadas y para todas las casuísticas explicadas en el sección 2.2.2.

\section{RESULTADOS}

En esta sección presentamos los resultados del Protocolo I y II. El objetivo del análisis que se presenta a continuación es determinar si la mejor ubicación del segundo sensor es C7 o determinar cómo influye la determinación de la posición neutral en la repetitividad de la medida.

\subsection{PROTOCOLO I}

En este primer protocolo, el sujeto realiza movimientos de forma independiente, calibrando una vez al comienzo de cada uno de ellos.

Las figuras 2 (T4) y 3 (C7) muestran los rangos medidos para los movimientos de flexión, extensión, inclinación izquierda, inclinación derecha, rotación izquierda, rotación derecha. Se representa la media y desviación estándar de la realización de los 36 sujetos que participaron en esta experimentación tanto para la primera sesión (Set 1), como para la segunda sesión (Set 2). Adicionalmente, se calcula el coeficiente ICC con el fin de estimar la repetitividad entre medidas en distintas sesiones con la misma población de sujetos.

\begin{tabular}{|c|c|c|c|}
\hline & $\begin{array}{c}\text { Set } 1 \text { (Mean } \pm \\
\text { SD) }\end{array}$ & $\begin{array}{c}\text { Set } 2 \text { (Mean } \pm \\
\text { SD) }\end{array}$ & ICC \\
\hline Flexion & $59,54^{\circ} \pm 14,14^{\circ}$ & $64,259 \pm 12,379$ & 0,75 \\
\hline Extension & $67,24^{\circ} \pm 14,32^{\circ}$ & $67,069 \pm 15,169$ & 0,95 \\
\hline $\begin{array}{c}\text { Right } \\
\text { lateral } \\
\text { inclination }\end{array}$ & $39,68^{\circ} \pm 7,85^{\circ}$ & $40,21^{\circ} \pm 6,37^{\circ}$ & 0,88 \\
\hline $\begin{array}{c}\text { Left } \\
\text { lateral } \\
\text { inclination }\end{array}$ & $43,560 \pm 8,019$ & 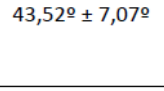 & 0,79 \\
\hline $\begin{array}{c}\text { Right } \\
\text { rotation }\end{array}$ & $73,27^{\circ} \pm 9,99^{\circ}$ & $75,51^{\circ} \pm 10,389$ & 0,91 \\
\hline $\begin{array}{c}\text { Left } \\
\text { rotation }\end{array}$ & $79,17^{\circ} \pm 10,75^{\circ}$ & $79,88^{\circ} \pm 10,579$ & 0,92 \\
\hline
\end{tabular}

Figura 2. Protocolo I. C0-T4

\begin{tabular}{|c|c|c|c|}
\hline & $\begin{array}{l}\text { Set } 1 \text { (Mean } \pm \\
\text { SD) }\end{array}$ & $\begin{array}{c}\text { Set } 2 \text { (Mean } \pm \\
\text { SD) }\end{array}$ & ICC \\
\hline Flexion & $51,71^{\circ} \pm 12,719$ & $52,74^{\circ} \pm 15,77^{\circ}$ & 0,88 \\
\hline Extension & $52,90^{\circ} \pm 14,280$ & $54,460 \pm 15,109$ & 0,93 \\
\hline $\begin{array}{c}\text { Right } \\
\text { lateral } \\
\text { inclination }\end{array}$ & $34,57^{\circ} \pm 6,41^{\circ}$ & $34,160 \pm 7,460$ & 0,88 \\
\hline $\begin{array}{c}\text { Left } \\
\text { lateral } \\
\text { inclination }\end{array}$ & $36,66^{\circ} \pm 7,77^{\circ}$ & $37,69^{\circ} \pm 7,72^{\circ}$ & 0,9 \\
\hline $\begin{array}{c}\text { Right } \\
\text { rotation }\end{array}$ & $64,760 \pm 71,150$ & $67,670 \pm 9,58^{\circ}$ & 0,9 \\
\hline $\begin{array}{c}\text { Left } \\
\text { rotation }\end{array}$ & $71,15^{\circ} \pm 7,98^{\circ}$ & $73,44^{\circ} \pm 11,77^{\circ}$ & 0,87 \\
\hline
\end{tabular}

Figura 3. Protocolo I. C0-C7

\subsection{PROTOCOLO II}

En el protocolo II los movimientos se realizan de forma complete, es decir, de extremo a extremo. La Figura 4 muestra los resultados para el Protocolo II para los movimientos de Flexo-Extensión (FE), Inclinación (LAT) y rotación (ROT) cuando el segundo sensor está ubicado en la vértebra T4.

\begin{tabular}{|c|c|c|c|}
\hline & Set 1 (Mean \pm SD) & Set 2 (Mean \pm SD) & ICC \\
\hline F-E & $121,91^{\circ} \pm 22,78 \circ$ & $121,77 \pm 19,98 \circ$ & 0,97 \\
\hline LAT & $80,42^{\circ} \pm 14,79 \circ$ & $79,46 \circ \pm 15,19 \circ$ & 0,91 \\
\hline ROT & $151,01^{\circ} \pm 17,31$ & $151,38 \pm 19,870$ & 0,96 \\
\hline
\end{tabular}

Figura 4. Medida del rango total para C0-T4 
La figura 5 muestra los mismos movimientos que en la figura anterior para la ubicación del segundo sensor en la vértebra $\mathrm{C} 7$.

\begin{tabular}{|c|c|c|c|}
\hline & Set 1 (Mean \pm SD) & Set 2 (Mean \pm SD) & ICC \\
\hline F-E & $101,06 \div \pm 21,58^{\circ}$ & $99,28 \div \pm 20,750$ & 0,98 \\
\hline LAT & $72,33^{\circ} \pm 13,160$ & $74,050 \pm 14,299$ & 0,93 \\
\hline ROT & $136,17^{\circ} \pm 20,22^{\circ}$ & $136,76 \circ \pm 19,470$ & 0,97 \\
\hline
\end{tabular}

Figura 5. Medida del rango total para $\mathrm{C} 0-\mathrm{C} 7$

Finalmente, la Figura 6 muestra los patrones normales del rango cervical (completos), obtenidos de la bibliografía, con el fin de que sirvan de comparativa con los resultados obtenidos en el Protocolo II.

\begin{tabular}{|c|c|c|}
\hline & Set 1 (Mean \pm SD) & Set 2 (Mean \pm SD) \\
\hline F-E & $131,7 \circ \pm 21,5 \circ$ & $132,8 \div \pm 20,5 \circ$ \\
\hline LAT & $88,4 \% \pm 17,9 \circ$ & $88,7 \circ \pm 18,19$ \\
\hline ROT & $148 \circ \pm 16,9 \circ$ & $148,3 \circ \pm 16,5 \circ$ \\
\hline
\end{tabular}

Figura 6. Rango total obtenido de la bibliografía (patrón de normalidad)

\section{DISCUSIÓN}

Tras evaluar los resultados obtenidos podemos concluir aspectos interesantes a tener en cuenta en el protocolo de medida del rango cervical empleando dos sensores inerciales. En primer lugar, consideramos como patrón normal del movimiento a aquel descrito en la Tabla I de la sección de Introducción. Una de las primeras conclusiones que podemos destacar es la amplia variabilidad en el patrón de normalidad definido en la bibliografía. De hecho, una justificación de ello lo podemos ver en los valores tan elevados de la desviación estándar. En nuestro caso, este valor también es, de hecho, elevado, al igual que en las publicaciones científicas citadas.

En relación a la comparativa entre el Protocolo I y el II, es preciso señalar que los ICCs son más bajos en el protocolo I (entre 0.75 y 0.92 para T4 y entre 0.87 y 0.93 para C7) que para el protocolo II (entre 0.91 y 0.97 ), como podemos ver en las figuras 2 y 3 frente a la 4 y la 5. Esto confirma que el protocolo II presenta una mejor repetitividad en la medida. En el caso del Protocolo II la diferencia de medida entre la sesión 1 y la sesión 2 es menor a un grado. Como se ha comentado, esto es particularmente importante si este tipo de sistemas se emplea para realizar el seguimiento de un tratamiento o una terapia.

En relación a la evaluación del mejor posicionamiento del segundo sensor en la vértebra
C7 o en la T4, podemos ver en primer lugar que el movimiento de extensión parece ser considerablemente menor (respecto a la normalidad) en todos los casos en los que el sensor se ubica en C7. Este hecho puede deberse principalmente a que el sujeto percibe el sensor en C7 en el movimiento de extensión y por tanto, no alcanza su máximo rango. Otra hipótesis es que se reduce el rango por artefactos que aparecen en la medida debido al movimiento de la piel.

Finalmente, centrándonos en el protocolo II, si comparamos la estimación del rango articular con el segundo sensor ubicado en T4 (Figura 4) observamos que es más próximo a los valores de normalidad (Tabla I) que con la ubicación del sensor en C7 (Figura 5). Por tanto, la ubicación en T4 parece ser una mejor opción, tal y como ya se había identificado en la literatura científica, [6].

\section{CONCLUSIÓN}

Este artículo ha estudiado dos factores que influyen significativamente en la precisión de la medida del rango articular cervical mediante tecnología inercial: la ubicación de los sensores en los segmentos corporales y la influencia de la posición neutral en la repetitividad de la medida.

Los resultados demuestran que se obtiene una estimación más precisa (más próxima a los valores de normalidad definidos en la bibliografía) del rango articular con la ubicación de un primer sensor en C0 (cabeza) y un segundo sensor en la vértebra T4 (en lugar de C7).

Por otro lado, los resultados demuestran que la correcta definición de la posición neutral antes de realizar la medida es fundamental para obtener una correcta repetitividad en la medida. Para reducir la variabilidad en la definición de la postura neutral, se aconseja la medida de rangos totales de movimiento, que corrigen tal variabilidad como ha quedado demostrado en la sección anterior.

\section{Agradecimientos}

Los autores desean agradecer la participación de alumnos y profesores de la Escuela Politécnica Superior de la Universidad CEU San Pablo que participaron en este estudio.

\section{Referencias}

[1] Michele Curatolo, MD,corresponding author Nikolai Bogduk, MD, Paul C. Ivancic, Samuel A. McLean, MD, Gunter P. Siegmund, and Beth Winkelstein. The role of tissue damage in whiplash associated disorder. Dec 2011 
[2] Sun Y. Yang, Anthony J. Boniello, Caroline E. Poorman, Andy L. Chang, Shenglin Wang, and Peter G. Passias. A Review of the Diagnosis and Treatment of Atlantoaxial Dislocations. May 222014

[3] Bax, M., Goldstein, M., Rosenbaum, P., Leviton, A., Paneth, N., Dan, B., ... Damiano, D. (2005). Proposed definition and classification of cerebral palsy. Developmental Medicine \& Child Neurology, 47(8), 571-576. http://doi.org/10.1017/S001216220500112X

[4] Fletcher James P., Bandy William D. Intrarater Reliability of CROM Measurement of Cervical Spine Active Range of Motion in Persons With and Without Neck Pain. Journal of orthopaedic \& sports physical therapy, October 2008

[5] Tousignant M., Smeesters C. Breton A., Corriveau H., Criterion Validity Study of the Cervical Range of Motion (CROM) Device for Rotational Range of Motion on Healthy Adults, November 2016

[6] Theobald et al. Do inertial sensors represent a viable method to reliably measure cervical spine range of motion?, Man Ther. 2012 Feb;17(1):92-6.doi:

10.1016/j.math.2011.06.007. Epub 2011 Jul 23. 